\title{
The oncological outcomes of small cell carcinoma of the bladder
}

Harley A. Williams, BSc ${ }^{1}$; Nahid Punjani, MD, $\mathrm{MPH}^{2}$; Obaidullah Khan, $\mathrm{MSc}^{3}$; Nicholas E. Power, MD, FRCSC ${ }^{2}$

${ }^{1}$ Department of Medical Biophysics, Schulich School of Medicine \& Dentistry, University of Western Ontario, London, ON, Canada; '2Department of Surgery, Division of Urology, University of Western Ontario, London, ON, Canada; ${ }^{3}$ Department of Medicine, Schulich School of Medicine \& Dentistry, University of Western Ontario, London, ON, Canada

Cite as: Can Urol Assoc J 2018 December 3; Epub ahead of print. http://dx.doi.org/10.5489/cuaj.5579

Published online December 3, 2018

$* * *$

\section{Abstract}

Introduction: Small cell carcinoma of the bladder (SmCC) is a rare and aggressive genitourinary malignancy. The paucity of clinical trials and outcome data provide no standard treatment guidelines. Accordingly, patient prognosis is poor. Our goal was to present the first comprehensive in-depth analysis of SmCC in a tertiary Canadian centre.

Methods: We retrospectively reviewed all patients diagnosed with primary SmCC at the London Regional Cancer Program between January 1990 and 2016. The primary outcome was overall survival (OS). We examined a number of secondary outcomes and baseline characteristics. Results: We identified 15 men and six women (median age 72 years) with a SmCC diagnosis (median followup 11.33 months). Median Charlson Comorbidity Index score was 7 (interquartile range [IQR] 5-10) and 15 patients had a smoking history. Most common presentation was gross hematuria (18 patients, 86\%), and pT2 stage at transurethral resection of the bladder tumour (TURBT) (n=7/21, 33\%), although five patients had cT4 (24\%). Pure SmCC was found in nine individuals (43\%), whereas 12 had mixed differentiation (57\%). From initial staging, 15 patients had extravesical disease (71\%), 10 had positive pelvic lymphadenopathy (48\%), and distant metastases occurred in six (29\%). In our series, five individuals (24\%) underwent cystectomy, 18 (86\%) received radiation, and 14 (67\%) received adjuvant chemotherapy. The median OS was 15 months (two-year OS was 19\%).

Conclusions: SmCC is a rare and aggressive form of bladder cancer. Despite multimodal therapy, prognosis remains guarded, with little improvement seen over the study's 25-year duration. An understanding of study limitations is warranted in interpretation of results. 


\section{Introduction}

Small cell carcinoma of the bladder is an extremely rare neoplasm, comprising less than $1 \%$ of all bladder malignancies. ${ }^{1}$ While uncommon, this neoplasm demonstrates aggressive behaviour and often has delayed presentation with advanced disease states, and is therefore associated with poor prognosis. ${ }^{2}$ With a predilection for males in their seventh or eighth decade compared to females, ${ }^{1,3}$ clinical presentation most often includes painless gross hematuria or obstructive urinary symptoms. Occasionally patient presentation can be accompanied by constitutional symptoms such as weight loss and fatigue. Differentation between small cell and urothelial bladder cancer can only be done through microscopic evaluation of tumor tissue. ${ }^{3}$ Furthermore, SmCC often presents in a mixed histologic pattern (40-70\%), ${ }^{3,4}$ most frequently with urothelial carcinoma (27\%), ${ }^{4}$ when compared to adenocarcinoma (3\%), ${ }^{4}$ or squamous cell $(1 \%),{ }^{4}$ and is rarely accompanied by sarcomatoid carcinoma. ${ }^{3}$

Unfortunately, since the first reported case of SmCC in 1981, very few reports from the English-language medical literature describe large series of patients. ${ }^{2,5}$ As a result of its rarity, the literature surrounding this neoplasm is sparse and treatment standards or guidelines have yet to be defined. Treatment typically involves a multimodal approach comprised of surgical or bladder-sparing intervention as well as chemotherapy and/or radiotherapy in both the neoadjuvant and adjuvant clinical settings. ${ }^{1,3}$ Limited studies have reported no survival improvement in individuals with nonmetastatic disease when treatment includes radical surgery and adjuvant therapy as opposed to chemotherapy and/or radiotherapy alone. ${ }^{4,6,7}$

Therefore, the purpose of this retrospective cohort study was to analyze the patient population and review characteristics in order to examine clinical outcomes, treatment modalities, cancer-free, and overall survival of all individuals diagnosed with pathologically confirmed small cell carcinoma of the bladder in a large Canadian series. We hypothesize that the cancerspecific and overall survival of patients diagnosed with small cell carcinoma of the bladder is dependent upon tumor stage at diagnosis. Our study aims to add to the relatively small body of current literature on small cell carcinoma of the bladder and hope that it will be of assistance in determining a better approach to the treatment of this malignancy. As the rarity of this tumor often precludes prospective clinical trial investigations, a better understanding of treatment paradigms could allow for improvements in long-term survival rates.

\section{Methods}

Upon receiving approval from the Western University Health Science Research Ethics Board and Lawson Health Research Institute, retrospective data was collected on all patients treated for small cell carcinoma of the bladder at the London Regional Cancer Program from January 1, 1990 to December 31, 2016. Individuals were identified using the International Classification for Diseases, Tenth Edition codes C67.0 - C67.9, which provided all individuals with a malignant neoplasm of the bladder. In total 21 patients, with biopsy proven SmCC of the bladder treated at our institution were identified using an institutional approved protocol to meet all 
inclusion criteria. We included all patients with complete data that were over the age of 18 . Individuals found to have small cell carcinoma of pure or mixed pathology were included in the study and comprise some of the 21 identified patients.

Information was collected from patient electronic and paper medical health records as well as London Regional Cancer Program's cancer registry. All subjects were assigned a study identification code which was documented in a secure and password protected file. All data manipulation and analysis was done with de-identified study identification codes. Data on patient demographics, clinical presentation, surgical findings, pathological characteristics, and laboratory investigations were recorded from patient medical records. This information was manually reviewed in order to identify patients which met out inclusion criteria.

Descriptive statistics were used to analyze the data. Disease-free and overall survival were reported using Kaplan-Meier curves as a function of time after diagnosis. Statistical analysis was completed using statistical software, Stata 14.1 (StataCorp LLC, Texas, USA). A $p$ value of less than 0.05 was considered to be statistically significant.

\section{Results}

A total of 21 patients were identified for analysis, 15 (71\%) of which were males, with a median follow-up of 11.33 months (IQR: 7.73-14.8). Demographic data can be seen in Table 1. In terms of age at diagnosis, 11 individuals (52\%) were diagnosed in the $7^{\text {th }}$ and $8^{\text {th }}$ decades of their life. Median age at diagnosis was 72 years old (IQR: 63-82). Race was not reported consistently enough to accurately characterize the study population. A large portion of the cohort had a BMI above 25, with 5 patients (24\%) considered obese and 8 patients (38\%) considered overweight. A history of smoking was also very prevalent in this cohort, with 12 patients being prior smokers and 3 patients being current smokers. The average pack-year history was 25.61 (IQR 1.25-43.75 pack-years). In terms of presenting symptoms, 18 individuals presented with gross hematuria, two of whom also had dysuria, while two others had dysuria with no gross hematuria. Frequency and urgency symptoms were seen in 6 individuals. Weight loss was observed in 6 patients. Urinary retention was seen in 3 patients.

With respect to history of cancer, 9 patients declared a personal history of cancer while 10 declared a family history of cancer. We also noted, quite interestingly, that 5 patients had a prior history of radiation therapy due to previous cancers. There was no clear pattern observed in the type of cancer in amongst those with either a personal or family history of cancer. In terms of pathology, 9 patients had histologically pure SmCC, while 12 were found to have mixed urothelial/squamous differentiation. The most common stage at diagnosis was pT2 (7 patients, 33\%), while 5 patients (24\%) had the cT4 stage. Extravesical disease was identified in 15 patients (71\%), while positive pelvic lymph nodes and distant metastases were identified in 10 patients (48\%) and 6 patients (29\%) respectively (Table 2). In terms of treatment, 5 patients (24\%) underwent cystectomy, 18 patients (86\%) received pelvic radiation (7 in the palliative setting), and 14 patients (67\%) had adjuvant platinum based chemotherapy. 11 of the 18 patients 
(61\%) who received pelvic radiation also received adjuvant platinum based chemotherapy. 3 of those $11(27 \%)$ received their radiation in a palliative setting. Of the 5 patients that underwent a cystectomy, multimodal treatment was provided in three cases (60\%). One received adjuvant chemotherapy and two received radiotherapy (1 in the palliative setting).

At the end of the study period, 19 of the 21 patients reviewed were deceased. The overall survival of patients with primary SmCC of the urinary bladder is shown in Figure 1. The median overall survival was 1.25 years (15 months), with no patient surviving longer than 41 months (3.4 yrs). The overall 1-year, 2-year, and 3-year survival rates were 57.9\%, 19.0\%, and 10.5\% respectively. Stratification by tumor stage is seen in Figure 2 which revealed no significant difference. Survival was further stratified by presence of distant metastasis as shown in Figure 3. None of the patients with M1 disease survived longer than 40 months (3.33 yrs). Pure SmCC and mixed SmCC were present in 9 patients (43.0\%) and 12 patients (57.0\%), respectively. No survival difference was observed between patients with pure SmCC and mixed SmCC tumors (Figure 4). Of the two patients alive at the end of the study period, both were considered to have pT2 disease at TURBT and received adjuvant platinum based chemotherapy with Etoposide. Additional agents included either Cisplatin or Carboplatin. The patient who received Etoposide and Carboplatin also received radiotherapy and had no evidence of disease at the last follow up timepoint (251 days, 0.69 yr. from chemotherapy end date). However, the patient who received a regimen of Etoposide and Cisplatin had evidence of a disease recurrence at the last follow up timepoint (198 days, 0.54 yr. from chemotherapy end date).

\section{Discussion}

Bladder cancer diagnoses constitute $4.6 \%$ of new cancer cases annually, and are the fifth leading cause of cancer-related deaths in the United States each year. ${ }^{8}$ Small cell carcinoma of the bladder, while comprising less than $1 \%$ of all bladder tumors, has a more aggressive behavior than typical genitourinary malignancies and results in a poorer patient prognosis and outcomes. ${ }^{2}$ Infrequently found within the genitourinary tract, small cell carcinomas have been reported within various other organ systems, most commonly in lung. ${ }^{9}$ While the exact pathogenesis of small cell bladder cancer is yet to be determined, SmCC is suspected to share the same histopathology as small cell carcinoma of the lungs (SCLC). ${ }^{9}$ There are currently no treatment guidelines for physicians that are considered best practice for this rare disease. ${ }^{10}$ Current treatment involves a multimodal approach which may include transurethral resections of bladder tumors (TURBT), chemotherapy, radiation, and/or radical cystectomy. ${ }^{1,3}$ The primary goal of our retrospective review was to analyze cancer specific and overall survival with respect to tumor stage, treatment prescribed, and tumor histology.

In previously reported series SmCC most often presented itself in patients of advanced age, specifically among individuals in their $7^{\text {th }}$ and $8^{\text {th }}$ decade, ${ }^{11}$ which we similarly noted in our series. In addition to age, a higher risk of disease development is associated with male biological sex, white race when compared to other-nonwhite races and current or prior cigarette use. ${ }^{9}$ Our 
study was in accordance with this, as approximately $71 \%$ of our cohort was male, resulting in a male to female ratio of 3:1. Additionally, $71 \%$ of the cohort either had a history of smoking or were smoking at the time of diagnosis. The effect of second hand smoke and toxic chemical exposure has yet to be determined; however, this variable was not assessed within our cohort. ${ }^{3}$ Presenting symptoms are similar to those of urothelial carcinoma and include but are not limited to gross hematuria, dysuria, frequency, and pelvic/suprapubic pain. ${ }^{9,12}$ Gross hematuria was by far the most prevalent symptom in our cohort with almost $86 \%$ of patients, followed by urinary frequency and urgency, seen in $29 \%$ of patients. Interestingly, other studies have illustrated dysuria as the second most common presenting symptom of SmCC, ${ }^{13,14}$ which was only seen in 4 patients (19\%) in our series. Furthermore, pain is often absent at the time of clinical presentation. ${ }^{1}$ In accordance with this, back, flank, and pelvic discomfort was only observed in 5 patients (24\%), all of which had high grade disease at diagnosis. Additionally, unexplained weight loss was present in $29 \%$ of the study cohort. Interestingly, amongst our cohort, the same percentage of patients presented with frequency/urgency and weight loss (6 patients, 29\%). Historically, in the literature, frequency/urgency is often identified in much greater proportions than weight loss. ${ }^{2,10,14,15}$

Many reports also indicate a higher incidence of mixed SmCC rather than pure SmCC. ${ }^{13,14,16,17}$ In fact, one study showed that $70 \%$ of their cohort had a mixed pathology. ${ }^{14}$ This trend was observed in our cohort with $57 \%$ of patients having mixed SmCC and urothelial carcinomas. While there is variability between the proportions of individuals presenting with mixed SmCC compared to Abrahams et al., there was no difference in overall survival between individuals with pure SmCC or SmCC of mixed histology. These results are consistent with other reports. ${ }^{2,14}$ Furthermore, tumor location was predominantly on the lateral wall of the bladder in our study, which is consistent with prior reviews. ${ }^{2,11,13,14,18-20}$

Consensus exists within the literature that SmCC most often presents at advanced stages (T3$\mathrm{T} 4 / \mathrm{N}+\mathrm{M}+)^{21}$ In our series, the most common stage at diagnosis was pT2 (33\%), however, 71\% of patients had pT2 disease or higher, $53 \%$ of which had at least pT3 disease. At the time of diagnosis, distant metastases were present in only six patients. A greater proportion of patients had positive pelvic lymph nodes (48\%) and extravesical disease (71\%). All individuals with metastatic disease at presentation did not undergo radical cystectomy but did receive a platinumbased chemotherapy regimen. Although long term survival is poor, others have confirmed the efficacy of platinum-based chemotherapy in the presence of stage IV disease. ${ }^{2,22-25}$ Additionally, radical cystectomy in the presence of metastatic disease is unlikely to provide a survivor benefit unless it is preceded by neo-adjuvant chemotherapy. ${ }^{2,6}$ Given that the individuals in our cohort with distant metastases did not undergo radical cystectomy, we were unable to assess if neoadjuvant platinum-based chemotherapy regimens did, in fact, yield a survivor benefit. While nevertheless, treatment approach does ultimately depend on clinical staging and patient performance status, chemotherapy is a key component of many multimodal approaches across all stages of SmCC disease. ${ }^{10,26}$ 
The use of radiotherapy alone in conjunction with bladder sparing approaches such as TURBT has shown to be associated with higher relapse rates and reduced long-term and median survival. ${ }^{11}$ However, the combined use of radiation and chemotherapy has shown to improve survival among individuals with SCLC. ${ }^{27}$ Furthermore, retrospective analysis has shown that concurrent chemotherapy and radiotherapy may be an effective bladder-sparing treatment approach, specifically in those who wish to delay or forgo radical cystectomy. ${ }^{15}$ In our cohort, of the five who underwent a radical cystectomy, radiation therapy was utilized in one case in an adjuvant setting, and two in a palliative setting. No survival benefit between those who received radiotherapy and those who did not was observed.

As previously mentioned, tumor stage at diagnosis has large implications on chosen treatment approach. In earlier stages, individuals may opt for surgical options which include bladder-sparing approaches, such as TURBT, or radical or partial cystectomy. ${ }^{10}$ While our study cohort was restricted by size, it is concerning that for patients who presented with nonmetastatic disease; no significant improvement in survival was observed when treatment involved radical surgery in conjunction with chemotherapy in both the neo-adjuvant and adjuvant clinical settings. With high rates of tumor recurrence, it would have been expected that radical cystectomy would provide the best strategy against subsequent malignancies, irrespective of the risk of morbidity associated with radical surgery. Furthermore, in a greater attempt to minimize the risk for disease recurrence, it would be expected that the administration of chemotherapy would provide an additive effect to overall survival rates. With disease presentation more common in later stages of life, it is imperative that the benefits of radical surgical options and chemotherapy, with respect to both overall survival and quality of life, outweigh the side effects of surgical- and/or drug-based intervention.

Although this study provides insight into the rarity of SmCC, it is important to point out its limitations. The retrospective nature of this study could have resulted in some level of selection bias. Additionally, minimal information was collected regarding the chemotherapy and radiation administered. Specifically, with respect to chemotherapy treatment, only duration and type was collected; modality, dose, frequency, and toxicity events, were neither reported nor analyzed. The use of radiation therapy was only collected in a dichotomous nature, no information regarding dosage/fractionation, duration, location, and intent were recorded. Our analysis focused on whether or not these treatments were used in patient care, as variability and inconsistency existed amongst the data available. We believe no significant differences in survival were observed due to our extremely small sample size. We continue to believe, that tumor stage at diagnosis not only dictates the course of future treatment, but also the duration of overall survival.

Despite these limitations, this study can be used to help generate future studies and hypotheses that aim to address important questions surrounding the treatment of SmCC. For instance, a better understanding of which factors dictate bladder-sparing approaches versus surgical intervention is warranted. Further prospective trials should focus on addressing the 
major questions surrounding the roles of chemotherapy, radiation, and surgical intervention in the optimal treatment of this disease. With the transition to electronic health records, it is important that focus is placed not only on case review of local tertiary care centers, but also on multicenter studies, with the hopes of improving patient prognosis of this extremely rare genitourinary malignancy. 


\section{References}

1. Soriano P, Navarro S, Gil M, Llombart-Bosch A. Small-cell carcinoma of the urinary bladder. A clinico-pathological study of ten cases. Virchows Arch. 2004;445:292-297. doi:10.1007/s00428-004-1041-1.

2. Choong NW, Quevedo JF, Kaur JS. Small cell carcinoma of the urinary bladder. The Mayo Clinic experience. Cancer. 2005;103(6):1172-1178. doi:10.1002/cncr.20903.

3. Celik O, Ekin G, Ipekci T, Budak S, Ilbey YO. Diagnosis and treatment in primary bladder small cell carcinoma: Literature review. Arch Ital di Urol e Androl. 2016;88(1):52-55. doi:10.4081/aiua.2016.1.52.

4. Pasquier D, Barney B, Sundar S, et al. Small cell carcinoma of the urinary bladder: A retrospective, multicenter rare cancer network study of 107 patients. Int J Radiat Oncol Biol Phys. 2015;92(4):904-910. doi:10.1016/j.ijrobp.2015.03.019.

5. Cramer SF, Aikawa M, Cebelin M. Neurosecretory granules in small cell invasive carcinoma of the urinary bladder. Cancer. 1981;47(4):724-730. doi:10.1002/10970142(19810215)47:4<724::AID-CNCR2820470417>3.0.CO;2-2.

6. Siefker-Radtke A, Dinney C, Abrahams N, et al. Evidence Supporting Preoperative Chemotherapy for Small Cell Carcinoma of the Bladder: a Retrospective Review of the M. D. Anderson Cancer Experience. J Urol. 2004;172(2):481-484. doi:10.1097/01.ju.0000132413.85866.fc.

7. Quek M, Nichols P, Yamzon J, et al. Radical Cystectomy for Primary Neuroendocrine Tumors of the Bladder: the University of Southern California Experience. J Urol. 2005;174(1):93-96. doi:10.1097/01.ju.0000162085.20043.1f.

8. Cancer of the Urinary Bladder - Cancer Stat Facts: National Cancer Institute. SEER Stat Fact Sheet.

9. Ismaili N. A rare bladder cancer--small cell carcinoma: review and update. Orphanet $J$ Rare Dis. 2011;6:75. doi:10.1186/1750-1172-6-75.

10. Thota S, Kistangari G, Daw H, Spiro T. A clinical review of small-cell carcinoma of the urinary bladder. Clin Genitourin Cancer. 2013;11(2):73-77. doi:10.1016/j.clgc.2012.11.002.

11. Church DN, Bahl A. Clinical review - Small cell carcinoma of the bladder. Cancer Treat Rev. 2006;32(8):588-593. doi:10.1016/j.ctrv.2006.07.013.

12. Moretto P, Wood L, Emmenegger U, et al. Management of small cell carcinoma of the bladder: Consensus guidelines from the Canadian Association of Genitourinary Medical Oncologists (CAGMO). Can Urol Assoc J. 2013;7(1-2):E44.

13. Blomjous CE, Vos W, De Voogt HJ, Van der Valk P, Meijer CJ. Small cell carcinoma of the urinary bladder. A clinicopathologic, morphometric, immunohistochemical, and ultrastructural study of 18 cases. Cancer. 1989;64(6):1347-1357. doi:10.1002/10970142(19890915)64:6<1347::AID-CNCR2820640629>3.0.CO;2-Q.

14. Abrahams NA, Moran C, Reyes AO, Siefker-Radtke A, Ayala AG. Small cell carcinoma of the bladder: A contemporary clinicopathological study of 51 cases. Histopathology. 2005;46(1):57-63. doi:10.1111/j.1365-2559.2004.01980.x. 
15. Lohrisch C, Murray N, Pickles T, Sullivan L. Small cell carcinoma of the bladder: long term outcome with integrated chemoradiation. Cancer. 1999;86(11):2346-2352. doi:10.1002/(SICI)1097-0142(19991201)86:11<2346::AID-CNCR24>3.0.CO;2-5.

16. Bex A, Nieuwenhuijzen JA, Kerst M, et al. Small cell carcinoma of bladder: A singlecenter prospective study of 25 cases treated in analogy to small cell lung cancer. Urology. 2005;65(2):295-299. doi:10.1016/j.urology.2004.09.049.

17. Bex A, De Vries R, Pos F, Kerst M, Horenblas S. Long-term survival after sequential chemoradiation for limited disease small cell carcinoma of the bladder. World J Urol. 2009;27:101-106. doi:10.1007/s00345-008-0304-x.

18. Holmäng S, Borghede G, Johansson SL. Primary small cell carcinoma of the bladder: a report of 25 cases. J Urol. 1995;153(6):1820-1822. http://www.ncbi.nlm.nih.gov/pubmed/7752326.

19. Lopez JI, Angulo JC, Flores N, Toledo JD. Small cell carcinoma of the urinary bladder. A clinicopathological study of six cases. Br J Urol. 1994;73(1):43-49. doi:10.1111/j.1464410X.1994.tb07454.x.

20. Mangar SA, Logue JP, Shanks JH, Cooper RA, Cowan RA, Wylie JP. Small-cell carcinoma of the urinary bladder: 10-year experience. Clin Oncol. 2004;16(8):523-527. doi:10.1016/j.clon.2004.06.024.

21. Ismaili N, Ghanem S, Mellas N, et al. Small cell carcinoma of the urinary bladder: a case report and review of the literature. J Cancer Res Ther. 2009;5(2):133.

22. Davis MP, Murthy MS, Simon J, Wise H, Minton JP. Successful management of small cell carcinoma of the bladder with cisplatin and etoposide. J Urol. 1989;142(3):817.

23. Mackey JR, Au HJ, Hugh J, Venner P. Genitourinary small cell carcinoma: determination of clinical and therapeutic factors associated with survival. J Urol. 1998;159(5):16241629. doi:10.1097/00005392-199805000-00058.

24. Angulo JC, Lopez JI, Sanchez-Chapado M, et al. Small cell carcinoma of the urinary bladder: a report of two cases with complete remission and a comprehensive literature review with emphasis on therapeutic decisions. J Urol Pathol. 1996;5:1-20.

25. Oesterling JE, Brendler CB, Burgers JK, Marshall FF, Epstein JI. Advanced small cell carcinoma of the bladder successful treatment with combined radical cystoprostatectomy and adjuvant methotrexate, vinblastine, doxorubicin, and cisplatin chemotherapy. Cancer. 1990;65(9):1928-1936. doi:10.1002/1097-0142(19900501)65:9<1928::AIDCNCR2820650910>3.0.CO;2-X.

26. Koay EJ, Teh BS, Paulino AC, Butler EB. Treatment trends and outcomes of small-cell carcinoma of the bladder. In: International Journal of Radiation Oncology Biology Physics. Vol 83. ; 2012:64-70. doi:10.1016/j.ijrobp.2011.05.039.

27. Pignon JP, Arriagada R, Ihde DC, et al. A meta-analysis of thoracic radiotherapy for small-cell lung cancer. N Engl J Med. 1992;327(23):1618-1624.

doi:10.1056/NEJM199212033272302. 
Figures and Tables

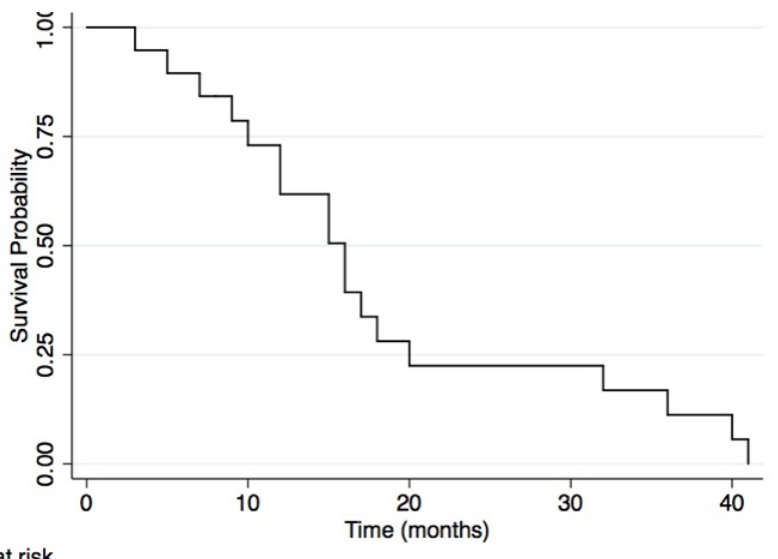

19

4

2
14
Fig.1. Kaplan-Meier curve for overall survival for patients with primary SmCC of the urinary bladder.

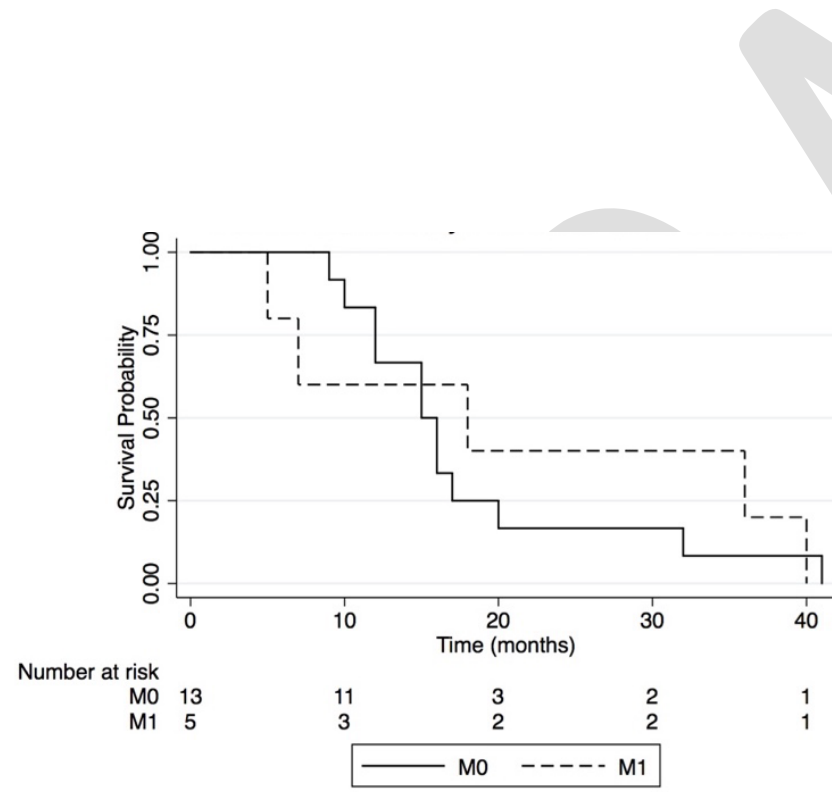

Fig. 3. Kaplan-Meier curve for overall survival, stratified by the presence (M1) or absence (M0) of metastasis at the time of diagnosis.

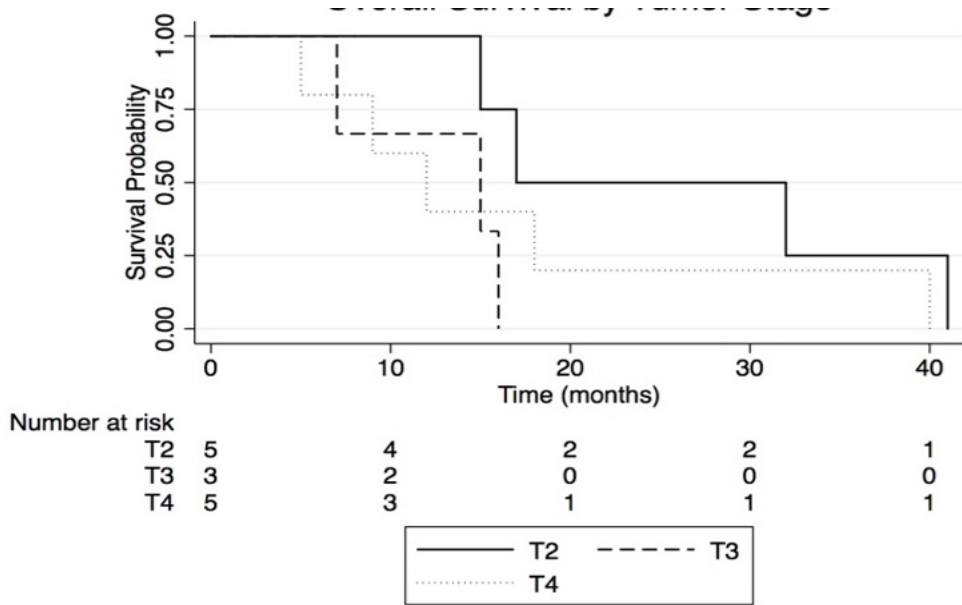

Fig.2. Kaplan-Meier curve for overall survival, stratified by TURBT stage according to the American Joint Committee on Cancer 2009 TMN classification system at time of diagnosis.

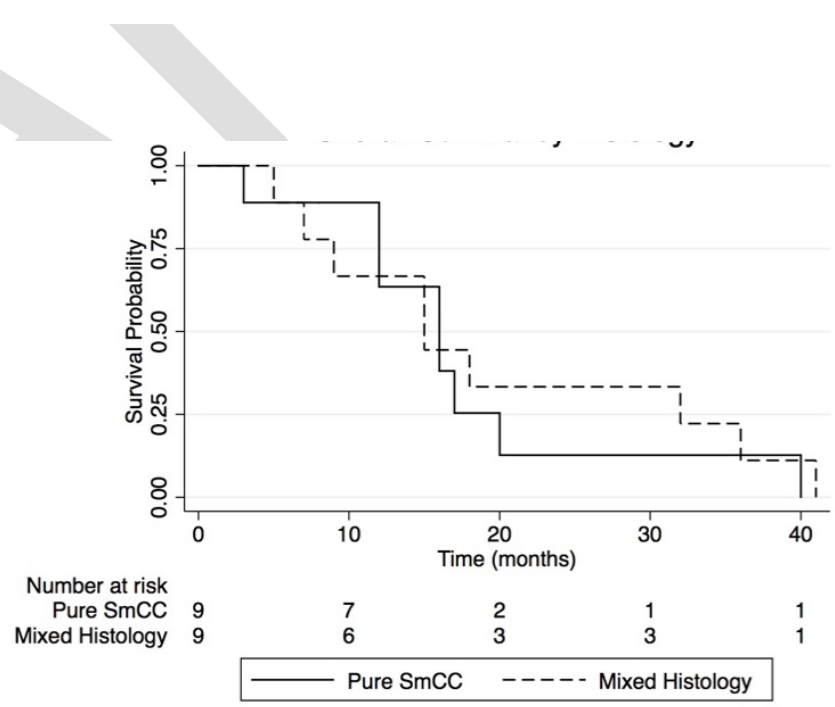

Fig. 4. Kaplan-Meier curve for overall survival, stratified by pure SmCC and mixed histology SmCC. 


\begin{tabular}{|c|c|c|}
\hline & $n=21$ & $\%$ \\
\hline $\begin{array}{l}\text { Age at diagnosis (yrs) } \\
\text { Median } \\
\text { IQR }\end{array}$ & $\begin{array}{c}72 \\
63-82\end{array}$ & \\
\hline $\begin{array}{l}\text { Gender } \\
\text { Male } \\
\text { Female } \\
\end{array}$ & $\begin{array}{c}15 \\
6 \\
\end{array}$ & $\begin{array}{l}71.4 \\
28.6 \\
\end{array}$ \\
\hline $\begin{array}{l}\text { BMI } \\
\quad \text { Median } \\
\text { IQR } \\
\end{array}$ & $\begin{array}{c}27.5 \\
24.9-30.4 \\
\end{array}$ & \\
\hline $\begin{array}{l}\text { Charlson comorbidity index } \\
\text { Median } \\
\text { IQR } \\
\end{array}$ & $\begin{array}{c}7 \\
5-10 \\
\end{array}$ & \\
\hline $\begin{array}{l}\text { Smoking status } \\
\text { Never } \\
\text { Current } \\
\text { Prior } \\
\end{array}$ & $\begin{array}{c}5 \\
3 \\
12 \\
\end{array}$ & $\begin{array}{l}25.0 \\
15.0 \\
60.0\end{array}$ \\
\hline $\begin{array}{l}\text { Symptoms } \\
\text { Gross hematuria } \\
\text { Frequency/urgency } \\
\text { Weight loss } \\
\text { Dysuria } \\
\text { Urinary retention } \\
\text { Incontinence } \\
\text { Pelvic pain } \\
\text { Back pain } \\
\text { Fatigue } \\
\text { Recurrent UTI } \\
\text { Flank pain } \\
\text { Left groin pressure } \\
\text { Clot retention } \\
\text { Intermittent chills } \\
\text { Sterile pyuria }\end{array}$ & $\begin{array}{l}18 \\
6 \\
6 \\
4 \\
3 \\
2 \\
2 \\
2 \\
2 \\
1 \\
1 \\
1 \\
1 \\
1 \\
1 \\
\end{array}$ & $\begin{array}{l}85.7 \\
28.6 \\
28.6 \\
19.0 \\
14.3 \\
9.5 \\
9.5 \\
9.5 \\
9.5 \\
4.8 \\
4.8 \\
4.8 \\
4.8 \\
4.8 \\
4.8 \\
\end{array}$ \\
\hline $\begin{array}{l}\text { Histological finding } \\
\text { SCC only } \\
\text { SCC with TCC/squamous }\end{array}$ & $\begin{array}{c}9 \\
12 \\
\end{array}$ & $\begin{array}{l}43.0 \\
57.0 \\
\end{array}$ \\
\hline $\begin{array}{l}\text { Stage, no. (\%) } \\
\text { pTa } \\
\text { pT1 } \\
\text { pT2 } \\
\text { pT3 } \\
\text { pT4 } \\
\text { Not available } \\
\end{array}$ & $\begin{array}{l}2 \\
0 \\
7 \\
3 \\
5 \\
4 \\
\end{array}$ & $\begin{array}{c}10.0 \\
0.0 \\
33.0 \\
14.0 \\
24.0 \\
19.0\end{array}$ \\
\hline
\end{tabular}


BMI: body mass index; IQR: interquartile range; SCC: small cell carcinoma, TCC: transitional cell carcinoma; UTI: urinary tract infection.

\begin{tabular}{|l|c|}
\hline \multicolumn{2}{|l|}{ Table 2. Initial sites of distance metastasis $(\mathbf{n}=\mathbf{6})$} \\
\hline Site & Frequency \\
\hline Regional Nodes & 4 \\
\hline Liver & 3 \\
\hline Lungs & 1 \\
\hline Prostate & 1 \\
\hline
\end{tabular}

